\title{
Tracking the Catalyst Layer Depth-Dependent Electrochemical Degradation of a Bimodal Pt/C Fuel Cell Catalyst: A Combined Operando Small- and Wide-Angle X-Ray Scattering Study
}

Johanna Schröder, ${ }^{a}$ Rebecca K. Pittkowski, ${ }^{\mathrm{a}, \mathrm{b}}$ Isaac Martens, ${ }^{\mathrm{c}}$ Raphael Chattot, ${ }^{\mathrm{c}}$ Jakub Drnec, ${ }^{\mathrm{c}}$ Jonathan Quinson, ${ }^{\mathrm{b}}$ Jacob J. K. Kirkensgaard, ${ }^{\mathrm{d}, \mathrm{e}}$ Matthias Arenz ${ }^{\mathrm{a}^{*}}$

a Department of Chemistry and Biochemistry, University of Bern, Freiestrasse 3, 3012 Bern, Switzerland

${ }^{\mathrm{b}}$ Department of Chemistry, University of Copenhagen, Universitetsparken 5, 2100 Copenhagen $\emptyset$, Denmark

${ }^{\mathrm{c}}$ European Synchrotron Radiation Facility (ESRF), 71 Avenue des Martyrs, 38000 Grenoble, France ${ }^{\mathrm{d}}$ Niels Bohr Institute, University of Copenhagen, Universitetsparken 5, 2100 København Ø, Denmark

e Department of Food Science, University of Copenhagen, Rolighedsvej 26, 1958 Frederiksberg, Denmark

*Corresponding author: matthias.arenz@dcb.unibe.ch

\begin{abstract}
The combination of operando small- and wide-angle X-ray scattering (SAXS, WAXS) in grazing incidence configuration is presented as a new approach to provide depth-dependent insights into the changes in mean particle sizes and phase fractions occurring for fuel cell catalysts during accelerated stress tests (ASTs). As fuel cell catalyst, a bimodal Pt/C catalyst was chosen that consists of two distinguishable particle size populations. The presence of the two different sizes should favor and uncover electrochemical Ostwald ripening as the major degradation mechanism, i.e., it is expected that the size of the larger particles in the Pt/C catalyst grows at the expense of the smaller particles. The grazing incidence mode performed at the European Synchrotron Radiation Facility (ESRF) at the ID31 beamline revealed an intertwinement of the depth dependent degradation. While the larger particles show the same particle size changes close to the electrolyte-catalyst interface and within the catalyst layer, for the smaller Pt nanoparticles a different degradation scenario is observed. At the electrolyte-catalyst interface, the smaller particles increase in size while their phase fraction decreases during the AST. However, in the inner catalyst layer the phase fraction of smaller particles increases instead of decreases. The results of a depth-dependent degradation strongly suggest to employ a depth-dependent catalyst design for future improvement of the catalyst stability.
\end{abstract}




\section{Keywords}

Small-angle X-ray scattering (SAXS); wide-angle X-ray scattering (WAXS); fuel cell catalyst degradation; accelerated stress test (AST); bimodal Pt/C catalyst

\section{Introduction}

Proton exchange membrane fuel cells (PEMFCs) offer a sustainable alternative to conventional combustion engines. ${ }^{1}$ Highly active precious metal based catalysts are typically used as state-of-theart, e.g., PtCo catalysts in the Mirai vehicle from Toyota. ${ }^{2}$ The translation of new PEMFC catalysts into their "real-life" application requires not only a high activity but also sufficient long-term catalyst stability of the costly catalyst. ${ }^{3,4}$ Therefore, stability investigations and suitable degradation protocols to simulate realistic application conditions recently received increasing attention. ${ }^{5-9}$ To reduce the time needed to induce catalyst degradation, accelerated stress tests (ASTs) are performed in fundamental screening studies. ${ }^{10-13}$ The performed ASTs can induce different electrochemical degradation mechanisms that affect the nanoparticle (NP) size of the degrading catalyst: (1) migration of particles followed by coalescence, (2) metal dissolution, (3) electrochemical Ostwald ripening, i.e., the growth of larger particles at the expense of smaller ones, and (4) particle detachment from the support. ${ }^{14,15}$ The particle size of fuel cell catalysts is commonly determined by (scanning) transmission electron microscopy ((S)TEM). To observe the change of some specific particles before and after the treatment, identical location (IL) (S)TEM is performed. ${ }^{16-24}$ By comparison, in smallangle X-ray scattering (SAXS) studies the particle size change can be investigated in larger volume as compared to the limited number of NPs and local characterization that is achieved by only (S)TEM. ${ }^{25-30}$ For example, ex situ SAXS was recently performed after applying AST protocols in a gas diffusion electrode (GDE) setup ${ }^{20,28,29}$ that eliminates mass transport limitations as compared to the rotating disk electrodes (RDEs) commonly used in fundamental research studies. ${ }^{31}$ However, both (S)TEM and SAXS were not clearly demonstrating the degradation mechanism of electrochemical Ostwald ripening which has been considered as one of the main degradation mechanisms in membrane electrode assemblies (MEA). ${ }^{5,10,32}$

In the present study, the depth-dependent (in the following also referred to as z-direction, see Figure S1) degradation within a catalyst layer was investigated by combining operando SAXS and wideangle X-ray scattering (WAXS). The aims were to reveal degradation phenomena such as Ostwald ripening as well as to probe if the degradation is homogeneous within the catalyst layer. To generate a system that should favor electrochemical Ostwald ripening, a catalyst with two distinguishable Pt

NP size distributions (bimodal catalyst) was prepared by mixing two commercial catalysts. ${ }^{33}$. Using 
an operando cell with grazing incidence configuration ${ }^{34}$ at the ID31 beamline at the European Synchrotron Radiation Facility (ESRF) in Grenoble, load cycle conditions of PEMFCs were simulated as AST following a protocol recommended by the Fuel Cell Commercialization Conference of Japan (FCCJ). ${ }^{12,35}$ In the separate analysis of operando SAXS and WAXS data, both techniques revealed different limitations in studying the change in size distribution of the bimodal catalyst. However, combining both SAXS and WAXS enabled determining the mean particle sizes as well as the respective phase fractions of the two populations. The mean particle sizes of both size populations were determined by SAXS, and those values were used as input for the Rietveld refinement of the WAXS data. Studying the degradation close to the electrolyte-catalyst interface, a phenomenon in line with Ostwald ripening is observed. However, investigation of the operando data in a middle depth within the catalyst layer unexpectedly revealed an increase of both the mean particle size and phase fraction of the smaller population. Based on the revealed depth-dependent catalyst degradation, a depth-dependent design of catalyst layers could be an important strategy to improve catalyst stability.

\section{Experimental section}

\subsection{Chemicals, materials, and gases}

For the catalyst ink formation, ultrapure Milli-Q water (resistivity $>18.2 \mathrm{M} \Omega \cdot \mathrm{cm}$, total organic carbon $(\mathrm{TOC})<5 \mathrm{ppb}$ ) from a Millipore system was used. Isopropanol (IPA, 99.7+ \%, Alfa Aesar), commercial Pt/C catalysts (TEC10E20A (1-2 nm Pt/C, $19.4 \mathrm{wt} \% \mathrm{Pt}$ ) and TEC10E50E-HT (4-5 nm $\mathrm{Pt} / \mathrm{C}, 50.6$ wt\% Pt), Tanaka kikinzoku kogyo), and Nafion dispersion (D1021, 10 wt. \%, EW 1100, Fuel Cell Store) were used for the preparation of the catalyst ink. The working electrolyte (WE) was prepared using a gas diffusion layers (GDL) with a microporous layer (MPL) on top (Freudenberg $\mathrm{H} 23 \mathrm{C} 8,0.230 \mu \mathrm{m}$ thick). In the electrochemical measurements diluted $70 \%$ perchloric acid $\left(\mathrm{HClO}_{4}\right.$, suprapur, Sigma Aldrich) as electrolyte and the gas argon (BIP ultrahigh purity, Air Liquide) were used. The two commercial Pt/C catalysts were dispersed in ethanol (absolute, VWR) and drop cast on holey $\mathrm{C}$ support film of $\mathrm{Cu} 300$ mesh grids (Quantifoil). The spectrochemical cell was cleaned by briefly immersing it in diluted Caro's acid at room temperature.

\subsection{Catalyst synthesis and ink formation}

The two commercial Pt/C catalysts (TEC10E20A and TEC10E50E-HT) were mixed in a Pt mass ratio of 0.4:0.6 to obtain the catalyst mixture with two size populations on different carbon $(\mathrm{C})$ flakes (bimodal Pt/C catalyst). In the following the two populations are referred to as the "smaller" and the "larger" population, respectively. The catalyst mixture was dispersed in a mixture of Milli-Q water 
and IPA (water/IPA volume ratio of 3:1) to prepare about $5 \mathrm{~mL}$ of ink (Pt concentration of $0.5 \mathrm{mg} / \mathrm{mL}$ ). To generate a suitable dispersion for further use, the mixture was sonicated for $5 \mathrm{~min}$ in a sonication bath (Bioblock Scientific, T 310/H, $35 \mathrm{kHz}$ ). After adding $38.5 \mu \mathrm{L}$ of Nafion (Nafion/carbon mass ratio of 1), the dispersion was again sonicated for $5 \mathrm{~min}$ in a sonication bath.

\subsection{Preparation of working electrode}

The Freudenberg GDL was placed between a glass funnel and a sand core filter in a vacuum filtration setup. As described by Yarlagadda et al. ${ }^{36}$ all this was placed on a collecting bottle. $4 \mathrm{~mL}$ of the prepared ink was diluted with $7 \mathrm{~mL}$ of Milli-Q water and $29 \mathrm{~mL}$ of IPA (water/IPA volume ratio of 1:3, Pt concentration of $0.05 \mathrm{mg} / \mathrm{L}$ ). After $1 \mathrm{~min}$ of sonication in a sonication bath, the diluted ink was filled in a funnel. The catalyst was deposited on top of the GDL using a diaphragm vacuum pump (Vacuubrand, MZ 2C, max. $1.7 \mathrm{~m}^{3} / \mathrm{h}$ and $9.0 \mathrm{mbar}$ ). The GDE was dried and stored in air. By this procedure, a nominal $\mathrm{Pt}$ loading of $0.208 \mathrm{mg} \mathrm{cm}^{-2}$ geo was generated. For the electrochemical measurement, the GDE after vacuum filtration was cut in circles with a diameter of $1 \mathrm{~cm}$.

\subsubsection{Electrochemical cell and measurement}

A transmission diffraction cell introduced before ${ }^{34}$ was used with $\mathrm{Ar}$ saturated $0.1 \mathrm{M} \mathrm{HClO}_{4}$ as electrolyte. The electrolyte was degassed in a bubbler before being introduced into the top of the cell, pumped out of the top of the cell, and recirculated back to the bubbler.

Activation of the catalyst was done by 10 cyclic voltammograms (CVs) between 0.06 and $1.0 \mathrm{~V}$ versus reversible hydrogen electrode $\left(\mathrm{V}_{\mathrm{RHE}}\right)$ using a scan rate of $50 \mathrm{mV} / \mathrm{s}$. ASTs applying potential steps between 0.6 and $1.0 \mathrm{~V}_{\mathrm{RHE}}(3 \mathrm{~s}$ holding per potential) simulating load-cycle conditions in a fuel cell vehicle were performed.

\subsection{Transmission electron microscopy (TEM)}

TEM was performed with a Jeol 2100 microscope, operated at $200 \mathrm{kV}$. At least five different areas of the TEM grid were imaged at a minimum of three different magnifications. The NP diameter was determined by measuring at least $200 \mathrm{NPs}$ with the image J software. The $\mathrm{Pt} / \mathrm{C}$ catalysts were dispersed in ethanol and drop cast onto Quantifoil grids.

\subsection{Small-angle X-ray scattering (SAXS)}

The SAXS and WAXS pattern were collected with a $77.0 \mathrm{keV}$ beam and reduced as described previously. ${ }^{37}$ The beam size was focused to approximately $5 \times 2 \mu \mathrm{m}$ (horizontal x vertical). In grazingincidence mode a depth profile was performed, see Figure S1 and S2 as an example. Three different 
depths were analyzed (referred to as depth 4, 8, and 12). Depth 4 was chosen as it is the first layer showing Pt pattern in WAXS, i.e., the layer closest to the electrolyte, and the SAXS pattern of depth 4 were analyzed using depth 3 as background. Depth 8 shows the maximum intensity in the SAXS diffraction i.e., the layer in the middle of the catalyst layer, and was analyzed using depth 18 as background. Depth 12 is deeper inside the catalyst layer and was analyzed using depth 18 as background.

An initial scan was performed before electrolyte was added to the cell. To ensure that the surface of the particles was fully reduced, the cell was held at $0.6 \mathrm{~V}_{\mathrm{RHE}}$ during X-ray data collection. Details about the measurement protocol during AST can be found in the SI, see Table S1.

The SAXS data analysis after background subtraction was performed as described previously ${ }^{27,29,38-}$ ${ }^{40}$ using a polydisperse spheres model. The radially averaged intensity $\mathrm{I}(\mathrm{q})$ is expressed as a function of the scattering vector $\mathrm{q}=4 \pi \cdot \sin (\theta) / \lambda$, where $\lambda$ is the wavelength and $2 \theta$ is the scattering angle. The background-corrected scattering data were fitted using a model of one or two polydisperse spheres described by a total volume distributed (i.e., volume-weighted) log-normal distribution. A hardsphere form factor $F\left(R_{2}, \eta_{2}\right)$ was used. ${ }^{41}$ The scattering data are fitted to the following expression:

$$
\mathrm{I}(\mathrm{q})=\mathrm{C}_{1} \cdot \int \mathrm{P}_{\mathrm{s} 1}(\mathrm{q}, \mathrm{R}) \mathrm{V}_{1}(\mathrm{R}) \mathrm{D}_{1}(\mathrm{R}) \mathrm{dR}+\mathrm{C}_{2} \cdot \mathrm{F}\left(\mathrm{R}_{2}, \eta_{2}\right) \int \mathrm{P}_{\mathrm{s} 2}(\mathrm{q}, \mathrm{R}) \mathrm{V}_{2}(\mathrm{R}) \mathrm{D}_{2}(\mathrm{R}) \mathrm{dR}
$$

$\mathrm{C}_{1}$ and $\mathrm{C}_{2}$ are scaling constants, $\mathrm{P}_{\mathrm{s} 1}$ and $\mathrm{P}_{\mathrm{s} 2}$ the sphere form factors, $\mathrm{V}_{1}$ and $\mathrm{V}_{2}$ the particle volumes and $D_{1}$ and $D_{2}$ the log-normal size distribution. The normalized sphere form factor is given by: ${ }^{42,43}$

$$
\mathrm{P}_{\mathrm{s}}(\mathrm{q}, \mathrm{R})=\left(3 \frac{\sin (\mathrm{qR})-\mathrm{qR} \cos (\mathrm{qR})}{(\mathrm{qR})^{3}}\right)^{2}
$$

and the log-normal distribution by:

$$
\mathrm{D}(\mathrm{R})=\frac{1}{\mathrm{R} \sigma \sqrt{2 \pi}} \exp \left(\frac{-\left[\ln \left(\frac{\mathrm{R}}{\mathrm{R}_{0}}\right)\right]^{2}}{2 \sigma^{2}}\right)
$$

where $\sigma$ is the variance and $\mathrm{R}_{0}$ (evaluated in $\AA$ ) the geometric mean of the log-normal distribution. The fitting was done using a home-written MATLAB code (available on request). The free parameters in the model are $C_{1}, R_{1}, \sigma_{1}, C_{2}, R_{2}, \sigma_{2}, \eta_{2}$. The values obtained for these parameters are reported in Table S2. To account for the two populations, the reported probability density functions were weighted by the relative surface contribution of the spheres (to point out the electrochemical contribution of the population) as detailed in the SI. Details about the determination of the mean particle sizes and deviation for the log-normal distributions are found in Table S2.

\subsection{Wide-angle $X$-ray scattering (WAXS)}


WAXS analysis was done by performing Rietveld refinement using GSAS- $\mathrm{II}^{44}$, as previously described $^{34}$. The structural model used for refinement consisted of two platinum $f c c$ phases (Fm-3m) of the same lattice constant $a$. The lattice constant was obtained from refinement with a single $f c c$ phase $(a=3.9046(6))$. The mean particle sizes obtained from SAXS were used as input parameter in the WAXS refinement as the volume-weighted average number of coherent scattering domains, which are calculated from the full-width-at-half-maximum of reflections refined with only Lorentzian line shapes. ${ }^{34}$ In this approach, we assume that no strain effects contribute to the broadening of reflections in the diffraction pattern. The contribution of the instrumental profile to broadening of the Bragg peaks can be neglected based on the refinement of the $\mathrm{CeO}_{2}$ calibrant: For the ceria NIST standard $(380 \mathrm{~nm})$, the instrumental profile contributes less than $10 \%$ to the width of the Bragg peaks. Therefore, no instrumental profile correction was included for the Rietveld refinement of the nanoparticles as the effect is insignificant compared to the peak broadening from the platinum nanoparticles and a complex model is further needed to account for the cell geometry. ${ }^{22}$ The background was described by a diffraction pattern from the grazing incident scan with minimal platinum content (depth 3 ) to describe the carbon background correctly.

The phase fraction is determined based on the crystallite sizes used as input values during the Rietveld refinement, which is not identical to the SAXS mean particle size. Therefore, we studied the influence of slight variations of the input values of the crystallite sizes. The input domain sizes were shifted by \pm 0.5 or $\pm 1.0 \mathrm{~nm}$ in three different ways in the WAXS refinement of the diffraction pattern of different depths, see Figure S7. First, the mean particle size of the larger population is changed by \pm 0.5 and \pm $1.0 \mathrm{~nm}$ while the size of the smaller population is kept constant. Second the size of the larger population is kept constant while the size of the smaller population is changed by $\pm 0.5 \mathrm{~nm}$. Third, the sizes of both populations are changed at the same time in the same direction by $\pm 0.5 \mathrm{~nm}$. The fractions obtained by varying the input domain sizes are found in the SI. These tests confirmed that the observed trend for the change in the two particle population fractions is not an artifact of using the input of mean particle sizes from the SAXS fitting as the crystallite size. Details about the WAXS measurement protocol during AST are found in the SI. 


\section{Results and discussion}

a)

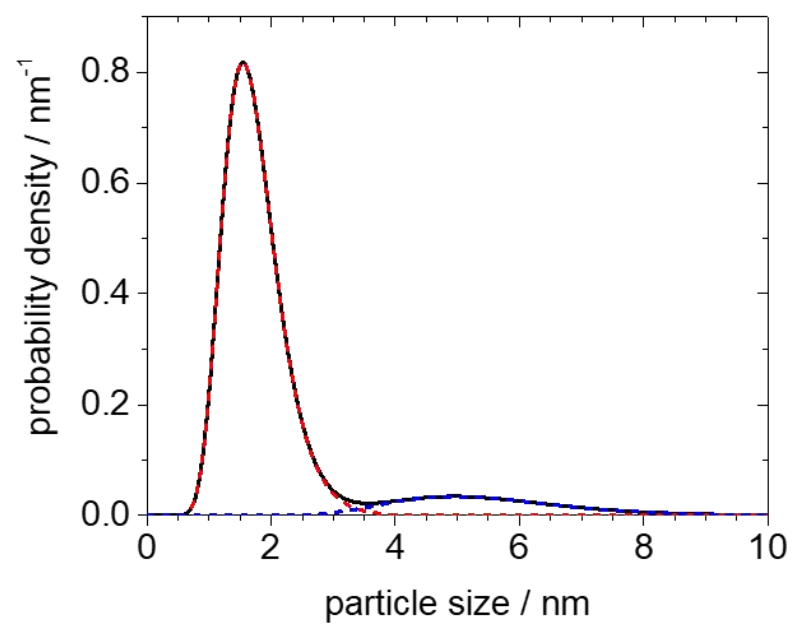

b)

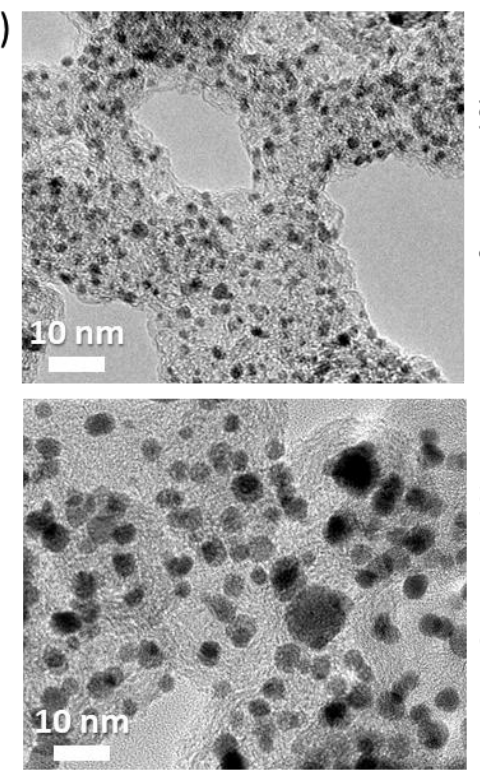

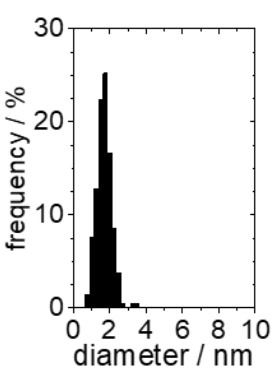

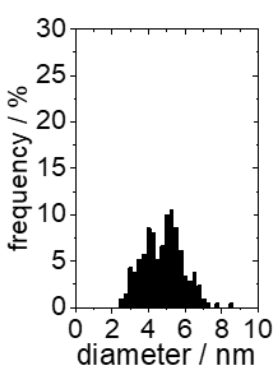

Figure 1. a) Calculated probability density function of the particle size of the as-prepared bimodal catalyst (black line) together with the probability densities of the separate size populations (dashed red and blue lines of the small and large size population, respectively) in a depth close to the electrolyte-catalyst interface derived from the SAXS data. b) TEM micrographs and size histograms of the two individual Pt/C catalysts that were mixed to obtain the bimodal Pt/C catalyst.

The aim of this work is to investigate the depth-dependent degradation mechanism(s) within a Pt/C fuel cell catalyst layer subjected to simulated load-cycle conditions. As main analytical tool, a combination of operando SAXS and WAXS was used to obtain precise information on the mean particle sizes and crystallite size fractions as a function of the duration of the AST protocol. To induce and uncover electrochemical Ostwald ripening, i.e., the growth of larger NPs at the expense of smaller ones, a bimodal $\mathrm{Pt} / \mathrm{C}$ catalyst obtained by mixing two different commercial $\mathrm{Pt} / \mathrm{C}$ catalysts with distinguishable size populations, was studied, see Figure 1. The here applied grazing-incidence mode allows a straightforward selection not only of different spots at the same depth (same x-y planes) in the catalyst layer, but additionally $\mathrm{z}$-scans can be performed ${ }^{45}$ to investigate if the degradation depends on the distance of the NPs to the electrolyte-catalyst interface, see Figure S1 for a scheme of the $\mathrm{x}-, \mathrm{y}-, \mathrm{z}$-directions relative to the electrolyte-catalyst interface.

Before discussing the influence of the AST protocol on the catalyst particles, we first introduce the combination of SAXS and WAXS analysis based on the pristine catalyst in a z-direction close to the electrolyte-catalyst interface, see grey scattering and diffraction data in Figure 2. Based on the fact that the bimodal $\mathrm{Pt} / \mathrm{C}$ catalyst is the result of mixing two individually characterized $\mathrm{Pt} / \mathrm{C}$ catalysts in 
known metal ratio (0.4:0.6 based on metal weight percentage of the two catalysts), the determined mean particle sizes and phase fractions in the bimodal $\mathrm{Pt} / \mathrm{C}$ catalysts can be compared to the "expected values". The analysis shows that the mean particle size of both populations of the bimodal catalyst can be determined by SAXS data analysis with high accuracy. However, a quantitative analysis of the relative number of NPs in the two size populations was difficult. The particle diameters and their standard deviations retrieved from the log-normal distributions obtained from the SAXS data are 1.7 \pm 0.5 and $5.5 \pm 1.5 \mathrm{~nm}$, see Figure 1a, Figure 3a, and Table S2 for details about the fitting parameters. Comparing the individual catalysts in Figure 1 with the prepared bimodal catalyst, it is demonstrated that the SAXS results are in good agreement with the TEM data of ca. 2 and ca. $5 \mathrm{~nm}$ average size. Although the expected relative number of NPs in the two size populations could be fitted to the SAXS data, in simulations the fit is ambiguous in this respect, i.e., no one-to-one correspondence between the intensities of the two populations of the same scattering data is found. However, this is not the case for the mean particle sizes, which remain the similar in simulations of different phase fractions of the two populations.

To address this limitation, we took advantage of WAXS data. The WAXS diffraction patterns clearly show the typical Bragg peaks of the platinum $f_{c c}$ phase which are convoluted with the signals of the carbon and polymer background at low $2 \theta$ angles, see Figure S2. From an angular range of six $2 \theta$ onwards, the platinum reflections are virtually free of background and can readily be analyzed by Rietveld refinement. ${ }^{45}$ Simultaneously varying both the fraction and crystallite size of the two populations of Pt NPs in the Rietveld refinement of WAXS data was not feasible due to the intertwining of these variables, as only one population with an average coherent domain size was obtained. Therefore, we used the mean particle size of the two populations derived by the SAXS fitting as pre-defined input values in the Rietveld refinement of the WAXS data. Two platinum phases of different coherent domain sizes (input of SAXS mean particle sizes) were used as the structural model in the Rietveld refinement. Although, the mean particle size derived from the SAXS fitting is not identical with the coherent domain size in diffraction, the two parameters are closely related. ${ }^{34}$ In this way the fractions of the platinum phases, i.e., their mass ratios, can be determined for the two size populations of Pt NPs. A representative refinement showing the two platinum phases of the structural model with different crystallite sizes is depicted in Figure S6. The obtained ratio of the smaller to the larger population of 0.44:0.56 in the pristine sample close to electrolyte-catalyst interface obtained from the combined SAXS and WAXS data analysis is in good agreement with the theoretical ratio of 0.4:0.6 expected from the sample preparation procedure, see Figure $3 \mathrm{~b}$. Thus, the combination of operando SAXS and WAXS simultaneously generates the data to readily evaluate 
both the size evolution of the two size populations and the fractions of the two size platinum crystallite domains of the bimodal Pt/C catalyst.

a)

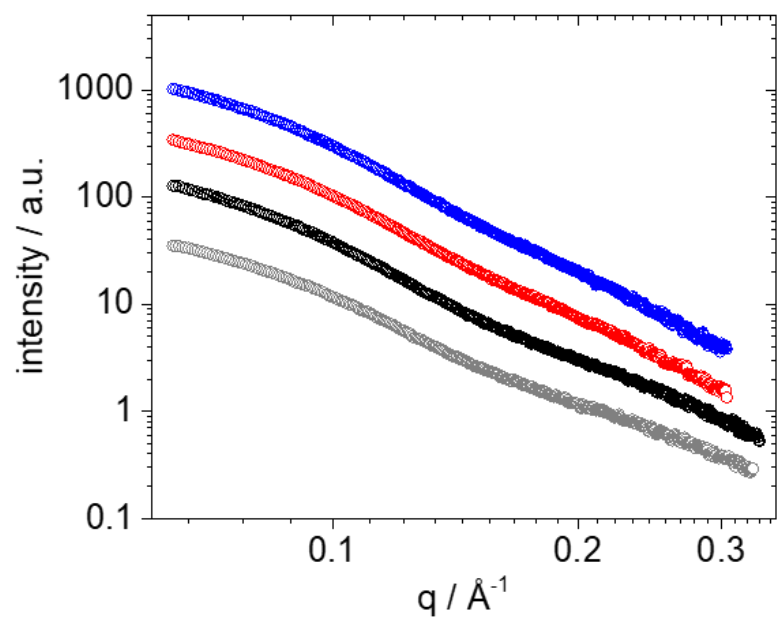

b)

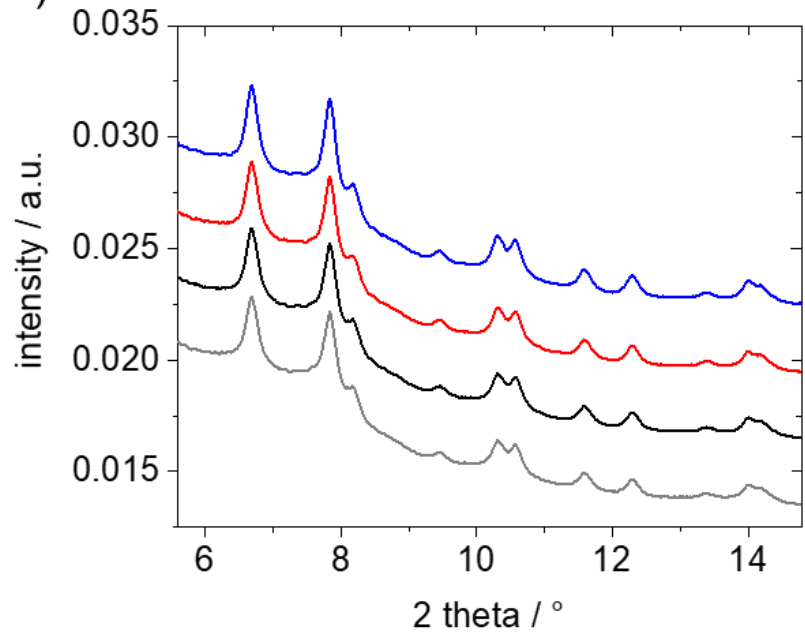

Figure 2. a) Background subtracted SAXS scattering data and b) WAXS diffractograms in an angular range from 5 to $15^{\circ} 2$ theta in the depth close to the electrolyte-catalyst interface: initial (grey), after catalyst cleaning (black), after 2500 AST cycles (red), after 5000 AST cycles (blue). The increase of the height and narrowing of the peaks of the diffractogram during the AST protocol imply an increase in particle size, which is in agreement with the mean particle size results. The data in a) and b) are shown with a vertical offset to improve visibility of the different datasets.

Before addressing the results on the influence of the AST protocol in detail, it should be mentioned that, at the beginning of an electrochemical experiment Pt/C catalysts typically need to be cleaned by cycling the electrode potential, see experimental section for details. Insertion of the catalyst into the operando cell $^{34}$, establishing electrochemical contact, and the applied cleaning procedure slightly affects the starting conditions. This effect can be seen by the fact that after electrolyte contact and the cleaning CVs the fraction of smaller NPs increases from 0.44:0.56 to 0.48:0.52 with respect to the larger size population, see Figure 3a, while the mean particle sizes remain relatively less affected as can be seen from the SAXS fitting, see Figure 3b. Such finding could be related to a pronounced Pt NP loss upon electrolyte contact during the cleaning CVs. Pt dissolution upon establishing electrochemical contact has been described by Pizzutilo et $a{ }^{8}{ }^{8}$ by coupling scanning flow cells (SFC) with inductively coupled plasma mass spectrometry (ICP-MS). Interestingly, here the change in phase fraction and the absence of changes in mean particle size of the two populations suggest a particle loss phenomenon with a higher loss of NPs in the large population. A preferential detachment of the larger particles would be in line with previous investigations by Mayrhofer et al. ${ }^{10}$ and Hartl et al. ${ }^{16}$ 
who demonstrated particle loss for the exact same catalyst that constitutes the larger fraction in the present work, see also discussion below. The periodically performed CVs, recorded after the AST treatment (see Table $\mathrm{S} 1$ for the related protocol) to determine the $\mathrm{H}_{\text {upd }}$ and to confirm electrochemical contact, however, did not influence the mean particle size; see overlap of the blue filled circles (after $\mathrm{CV}$ ) and the blue circles in Figure 3a. Therefore, in the following all changes observed during the measurements are assigned to the applied AST protocol.

The effect of the AST treatment on the catalyst is analyzed first at a depth close to the electrolytecatalyst interface. Representative operando SAXS and WAXS data of the initial Pt/C catalyst, after catalyst cleaning, after 2500, and after 5000 AST cycles are shown in Figure 2. Based on the mean particle size changes obtained from the SAXS data (see Figure S4 and Table S2) three main observations are made, see Figure 3a. First, both populations of the catalyst located in the catalyst layer close to the electrolyte-catalyst interface increase in size. Second, at the beginning of the AST, the increase in size per treatment cycle is more pronounced than at the end of the AST. Third, the increase in size of the population of the larger particles is less pronounced (from $5.48 \pm 1.45$ to 5.87 $\pm 1.61 \mathrm{~nm}$, size increase of around $7 \%)$ than the size increase of the smaller particles $(1.76 \pm 0.48$ to $2.42 \pm 0.79$, size increase of around $50 \%$ ), see Figure $3 \mathrm{a}$. The observation of a more pronounced size increase at the beginning of the AST as compared to that during the AST is in line with previous findings. ${ }^{3,8,18}$ But the finding that both distributions increase in size is not in obvious agreement with an electrochemical Ostwald ripening process. In particular, when Ostwald ripening would occur between the two size populations, a homogeneous dissolution of Pt NPs in the smaller population could be expected to lead to a decrease in the mean particle size of this population. Hence, the observed behavior is more in line with a preferential dissolution of smaller Pt NPs within the small population thus shifting the average particle size to larger values. The increase in average particle size of the large population then can be explained by either electrochemical Ostwald ripening or the same phenomena, i.e., preferential dissolution of smaller NPs within the large population. So, dissolution of the smaller particles followed by Ostwald ripening seems to occur within but not between both populations. As a consequence, Ostwald ripening is a very local process in the nanometer scale that does not include pronounced redeposition of dissolved Pt ions on neighboring catalyst flakes of the two different catalysts. Such mechanism would be an agreement with the Pt species being mobile on the carbon support as previously proposed by Ferreira et al. ${ }^{5}$ Diffusion of soluble Pt species on a larger scale does not lead to redeposition and thus Ostwald ripening, however, in an MEA environment Pt precipitation can occur due to hydrogen crossover.

If there would be a substantial Ostwald ripening process between the two populations, the relative number of particles in the two populations should dramatically change as a result of the AST treatment. 
Analyzing the relative number of particles in the two populations, i.e., the phase fraction, it is seen that indeed the phase fraction of the smaller size population decreases when applying the AST protocol. The relatively small change in phase fraction, however, suggests that the observed effect is more likely the sole result of a particle size dependent Pt dissolution. Additionally, during the AST protocol, preferential NP dissolution within both populations leads to an increase in the mean particle size. Due to the size difference of both populations, the absolute number of particles in the small population decreases with respect to the large population.

Trying to understand the depth dependence of the degradation mechanism, the changes of the particle size and the phase fraction were also analyzed in a "middle depth" with respect to the electrolytecatalyst interface.

a)

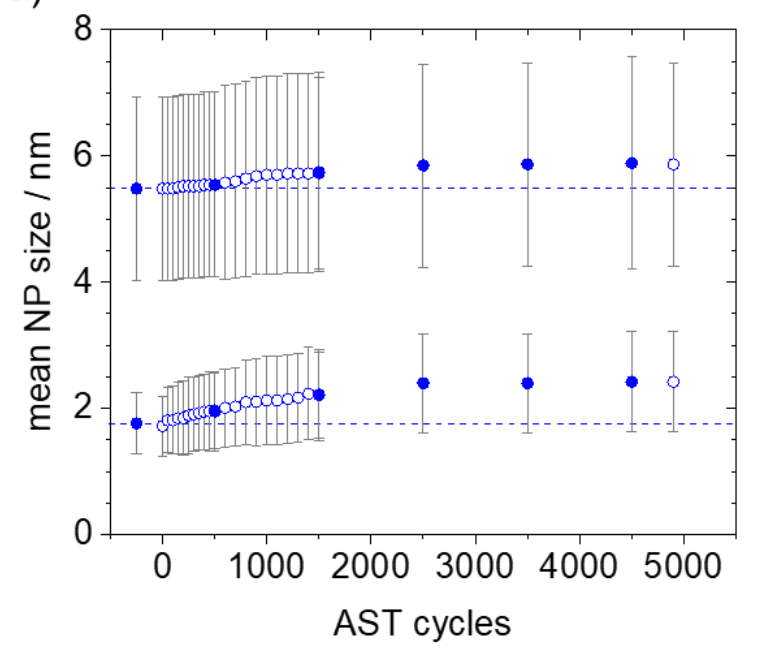

b)

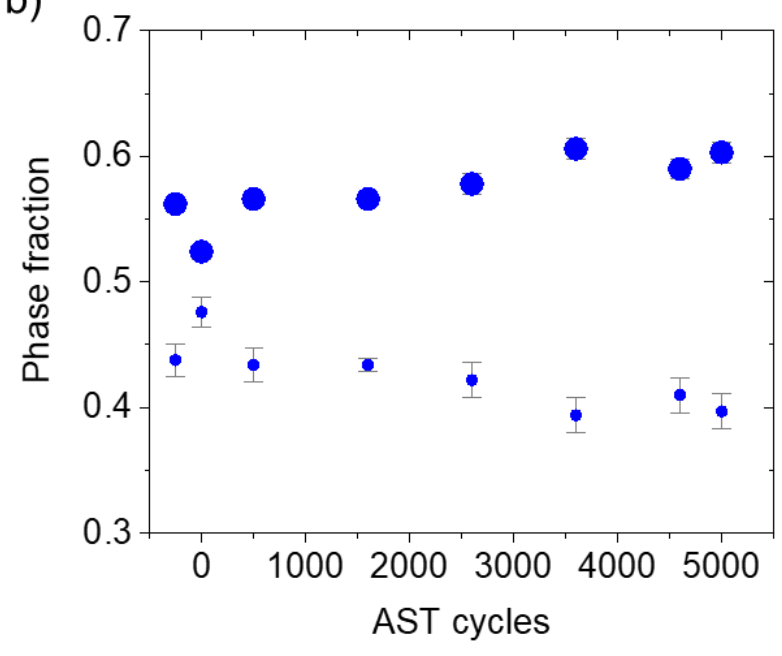

Figure 3. a) Mean particle size of the bimodal Pt NP/C catalyst in the depth close to the electrolytecatalyst interface plotted together with the standard deviation of a log-normal distribution applying load-cycle conditions as AST protocol in an operando cell after the AST cycles (blue open circles) and after performed CVs (blue filled cycles). b) Phase fractions of the two size populations (small and large circles, for the smaller and larger size populations, respectively) obtained from Rietveld refinement of in the depth close to electrolyte-catalyst interface, keeping the particle sizes determined via SAXS constant. The data points of the pristine sample are given on the $\mathrm{x}$-axis for a negative AST number.

It is seen that in both depths (close to the electrolyte-catalyst interface and in the middle catalyst depth) the mean particle size of both populations increases with the AST treatment. This confirms that the complete catalyst layer is electrochemically active and thus connected, see Figure 4a. Based 
on the observations we can identify four common characteristics for the two depths analyzed. First, the particle size and the size change during the AST of the larger population are similar in the two depths. Second, the particle size of the smaller size population increases in a more pronounced way than the particle size of the larger population (from $2.43 \pm 0.67$ to $3.52 \pm 0.68 \mathrm{~nm}$ (size increase of around $45 \%$ ) and from $5.50 \pm 1.17$ to $5.99 \pm 1.15 \mathrm{~nm}$ (size increase of around $9 \%$ ) in the middle catalyst layer depth). Third, the relatively size increases of the two population are comparable in the depth close to the electrolyte-catalyst interface (7 and $50 \%$ for the small and large population, see blue circles in Figure 4a) or in the middle of the catalyst layer ( 9 and $45 \%$, see black circles). Fourth, the particle size change of the larger population seems to be independent of the depth. However, there is also a distinct difference between the two depths. Close to the electrolyte interface, the smaller population is characterized by a slightly smaller particle size than the smaller population in the middle depth. This size difference is apparent already before the start of the AST protocol. On the contrary, comparing the scattering data at two different positions of the same depth in the middle of the catalyst layer in Figure S5, it is confirmed that these observations are representative for the macroscopic catalyst layer, i.e., the changes in particle size of the two populations are identical at both $x-y$ positions in the layer.

The obtained ratio of the smaller to the larger population of 0.46:0.54 in the pristine sample in the middle of the catalyst layer is comparable to the theoretical ratio of 0.4:0.6. Again, after electrolyte contact and the cleaning CVs, the fraction of the smaller NPs increases to 0.51:0.49 with respect to the larger size population, see Figure 4b, while the mean particle sizes remain unchanged as can be seen from the SAXS fitting, see Figure 4a. This phenomenon is similar to that observed in the depth close to the electrolyte-catalyst interface, see Figure 3a und b. However, applying the AST, the phenomenon of an increase in the fraction of smaller NPs continues during further AST cycles. It is found that the fraction of the smaller as compared to the larger population in the middle of the catalyst layer continuously increases to reach a value of 0.6:0.4 at the end of treatment, see Figure $3 \mathrm{c}$. Concomitantly, the particle sizes in both populations increase, see Figure $3 \mathrm{a}$.

This behavior of the $\mathrm{Pt} / \mathrm{C}$ catalyst in the middle of the catalyst layer is unexpected. It would be more intuitive to observe a growth of the NPs and a decrease of the smaller NP fraction, e.g., when Ostwald ripening is the main degradation process. Therefore, we analyzed additional data obtained from different z-directions at a third depth closer to the GDL, i.e., deeper inside the catalyst layer. The analysis of the SAXS and WAXS data in the depth far away from the electrolyte-catalyst interface show the same unexpected results in change of the mean particle sizes and the phase fractions as observed in the middle catalyst layer, see Figure 4a and c. To explain those observations, several scenarios can be considered. An enhanced dissolution of the larger particles seems to be unlikely. In 
addition, a migration of smaller particles from the upper catalyst layers could explain the observation but seems unlikely as well. However, a preferential particle detachment due to support oxidation as documented before of the same catalyst with larger particles ${ }^{10,16}$ could explain the findings, see Figure 5.

a)

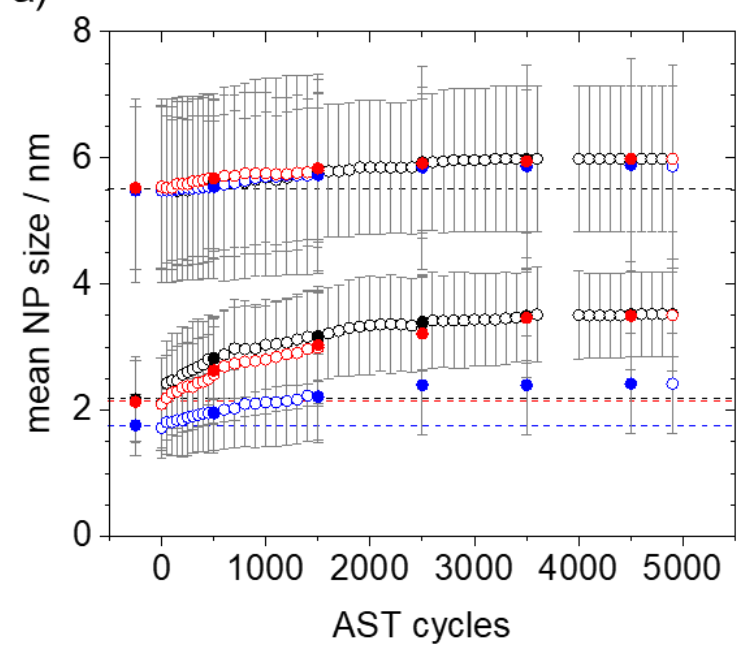

b)

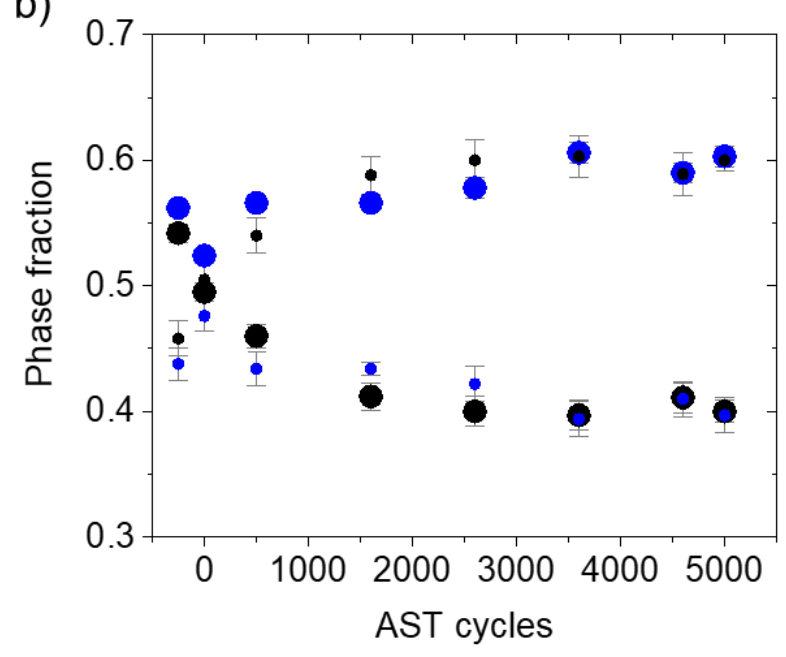

c)

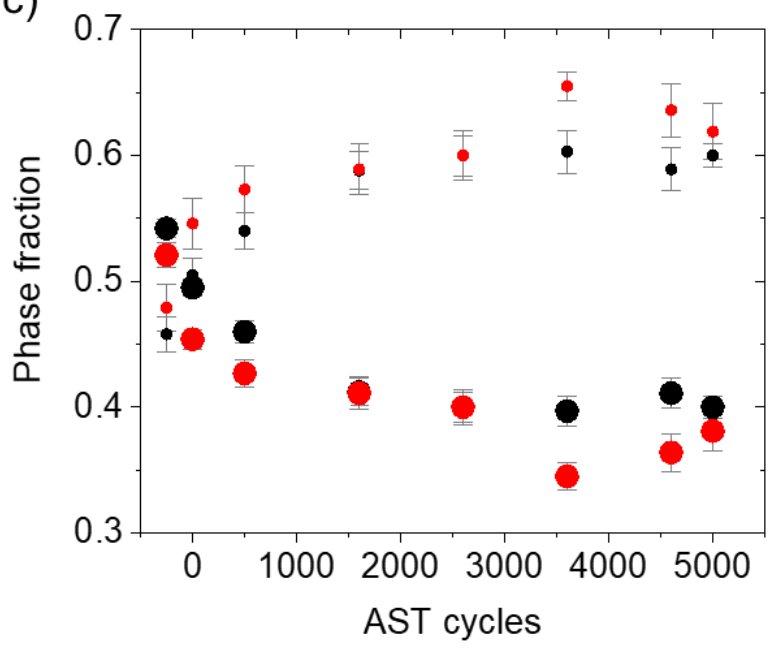

Figure 4. a) Mean particle size of the bimodal Pt NP/C catalyst plotted together with the standard deviation of a log-normal distribution applying load-cycles conditions as AST protocol in an operando cell after the AST cycles (empty circles) and after CVs (filled circles) close to the electrolyte (blue), in the middle depth (black), and deeper inside the catalyst layer (red). Phase fractions of the two size populations (small and large circles, for the smaller and larger size populations, respectively) obtained from Rietveld refinement of the different depth keeping the particle sizes determined via SAXS constant, b) close to the electrolyte (blue) and in the middle catalyst layer (black), c) in the middle (black) and deeper inside the catalyst layer (red) (the red filled circles are placed in front of the red circles, due to an overlap the black circles are sometimes covered). The data points of the pristine sample are given on the $\mathrm{x}$-axis for a negative AST number. 
In this context, it is important to point out that refinement of the diffraction pattern yields a relative and not an absolute number for both populations. Hence, the increase in the fraction of the smaller population could also be explained by a loss of the larger particles. Such a particle detachment of larger particles followed by dissolution but without redeposition was observed before by Mayrhofer et $a l .{ }^{10}$ and Hartl et $a l .{ }^{16}$ by performing IL-TEM in an RDE setup. Stronger support-particle interaction of the larger particles due to the larger contact area with the support, as compared to smaller ones, could indicate that larger particles interact more strongly with the support and might migrate and detach less. ${ }^{46}$ However, the support-particle interaction is determined by other factors, e.g., by the degree of graphitization of the carbon support. ${ }^{47}$ The observed preferential particle detachment of larger NPs indicates a degradation scenario based on the support-particle interaction being a combination of both the nature of particles and support. Assuming such a possible detachment of the larger NPs inside the catalyst layer, the NPs have a certain probability to redeposit onto the support during their pathway through the pores of the catalyst layer. Hence, the number of larger particles inside the catalyst layer is decreasing while it is increasing closer to the electrolyte, see Figure 5.

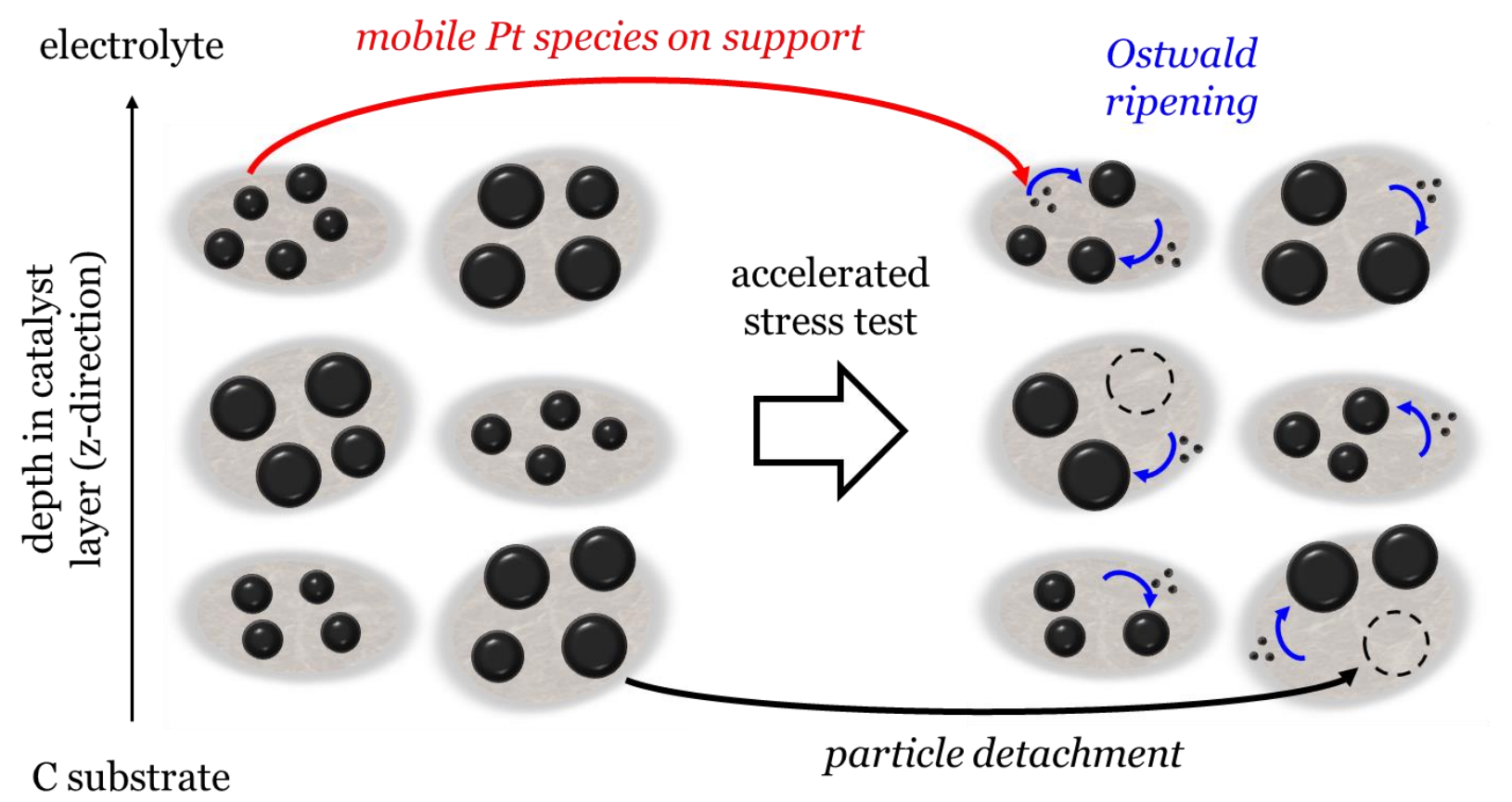

Figure 5. Scheme of the proposed depth-dependent degradation mechanism in the $\mathrm{Pt} / \mathrm{C}$ catalyst.

\section{Conclusion}


The combination of operando SAXS and WAXS in grazing incidence configuration is used to investigate catalyst layer depth-dependent degradation of a fuel cell catalyst subjected to ASTs. A bimodal $\mathrm{Pt} / \mathrm{C}$ catalyst was chosen that consists of two distinguishable particle size populations to maximize the chance to observe the expected electrochemical Ostwald ripening where the size of the larger particles in the $\mathrm{Pt} / \mathrm{C}$ catalyst grows at the expense of the smaller particles. However, it is seen that the degradation mechanism is more complex. The degradation of the $\mathrm{Pt} / \mathrm{C}$ catalyst close to the catalyst-electrolyte layer shows features that can be associated with preferential dissolution of small Pt particles and potentially with Ostwald ripening via Pt species mobile on the carbon support. Starting the AST, the average particle size of the smaller population slightly increases while the phase fraction decreases during the AST. The increase in average size indicates that within the population smaller particles preferentially dissolve and $\mathrm{Pt}$ species redeposit on the nanometer scale, the decrease in phase fraction indicates that dissolution is more pronounced in the small population.

However, in the inner catalyst layer, the phase fraction of the smaller particles increases instead. Furthermore, the mean particle size of the small population is larger at the inner catalyst layer. By comparison, the mean particle size of the larger population seems homogenous across the catalyst layer and in all cases a slight increase in size is observed during the AST. Although the increase in phase fraction of the smaller particles is difficult to explain at this point, the results show that the degradation of the $\mathrm{Pt} / \mathrm{C}$ catalyst within the layers is far from being homogeneous. This raises the question if the liquid electrolyte has any influence on the observed mechanism. Interestingly, it is seen that contacting the catalyst with the electrolyte and applying cleaning cycles, the same increase in phase fraction of the smaller particles is observed. It can be speculated that the contact of the electrolyte leads to a pronounced particle loss mechanism, as has previously been observed. In any case, the fact that the observed change in phase fraction is not associated with an increase in mean size suggests that this degradation process is not intrinsically size-dependent but more related to the different catalyst synthesis procedures of both catalysts, in particular - the 'HT' from the commercial name implied - heat treatment (TEC10E50E-HT) of the larger population may lead to a pronounced degradation upon contact to the electrolyte and by applying cleaning cycles.

In conclusion, the results suggest that Ostwald ripening is a nanoscale phenomenon most likely triggered by Pt species mobile on the carbon support. Longer scale phenomena in an MEA are related to Pt reduction via hydrogen cross-over. A significant contribution to the degradation, however, is also related to the anchoring of the nanoparticles on the support. Both effects seem to have different dependences on the distance to the solid-liquid interface. The combination of operando SAXS and WAXS in grazing incidence configuration is a powerful tool that also could be used to study new degradation inhibition strategies such as the design of gradient composition and particle size 
distributions. Furthermore, the approach demonstrated here can be easily transferred to mechanistic studies of catalyst systems with two or more size populations consisting of different metal particles.

\section{Acknowledgements}

This work was supported by the Swiss National Science Foundation (SNSF) via the project No. 200021_184742 and the Danish National Research Foundation Center for High Entropy Alloy Catalysis (CHEAC) DNRF-149. S. B. Simonsen and L. Theil Kuhn, Technical University of Denmark, are thanked for access to TEM. The authors also thank ESRF for beamtime at ID31 beamline and $\mathrm{H}$. Isern and F. Russelo for technical support.

\section{References}

(1) Pollet, B. G.; Kocha, S. S.; Staffell, I. Current Status of Automotive Fuel Cells for Sustainable Transport. Current Opinion in Electrochemistry. Elsevier August 1, 2019, pp 9095. https://doi.org/10.1016/j.coelec.2019.04.021.

(2) Yoshida, T.; Kojima, K. Toyota MIRAI Fuel Cell Vehicle and Progress Toward a Future Hydrogen Society. Electrochem. Soc. Interface 2015, 24 (2), 45-49. https://doi.org/10.1149/2.F03152if.

(3) Marcu, A.; Toth, G.; Kundu, S.; Colmenares, L. C.; Behm, R. J. Ex Situ Testing Method to Characterize Cathode Catalysts Degradation under Simulated Start-up/Shut-down Conditions - A Contribution to Polymer Electrolyte Membrane Fuel Cell Benchmarking. J. Power Sources 2012, 215, 266-273. https://doi.org/10.1016/j.jpowsour.2012.05.010.

(4) Katsounaros, I.; Cherevko, S.; Zeradjanin, A. R.; Mayrhofer, K. J. J. J. Oxygen Electrochemistry as a Cornerstone for Sustainable Energy Conversion. Angew. Chemie - Int. Ed. 2014, 53 (1), 102-121. https://doi.org/10.1002/anie.201306588.

(5) Ferreira, P. J.; la O’, G. J.; Shao-Horn, Y.; Morgan, D.; Makharia, R.; Kocha, S.; Gasteiger, H. A.; La O', G. J.; Shao-Horn, Y.; Morgan, D.; Makharia, R.; Kocha, S.; Gasteiger, H. A. Instability of Pt/C Electrocatalysts in Proton Exchange Membrane Fuel Cells. J. Electrochem. Soc. 2005, 152 (11), A2256. https://doi.org/10.1149/1.2050347.

(6) Borup, R.; Meyers, J.; Pivovar, B.; Kim, Y. S.; Mukundan, R.; Garland, N.; Myers, D.; Wilson, M.; Garzon, F.; Wood, D.; Zelenay, P.; More, K.; Stroh, K.; Zawodzinski, T.; Boncella, J.; McGrath, J. E.; Inaba, M.; Miyatake, K.; Hori, M.; Ota, K.; Ogumi, Z.; Miyata, S.; Nishikata, A.; Siroma, Z.; Uchimoto, Y.; Yasuda, K.; Kimijima, K. I.; Iwashita, N. Scientific Aspects of Polymer Electrolyte Fuel Cell Durability and Degradation. Chemical Reviews. American Chemical Society October 2007, pp 3904-3951. 
https://doi.org/10.1021/cr0501821.

(7) Castanheira, L.; Silva, W. O.; Lima, F. H. B. B.; Crisci, A.; Dubau, L.; Maillard, F. Carbon Corrosion in Proton-Exchange Membrane Fuel Cells: Effect of the Carbon Structure, the Degradation Protocol, and the Gas Atmosphere. ACS Catal. 2015, 5 (4), 2184-2194. https://doi.org/10.1021/cs501973j.

(8) Pizzutilo, E.; Geiger, S.; Grote, J.-P.; Mingers, A.; Mayrhofer, K. J. J.; Arenz, M.; Cherevko, S. On the Need of Improved Accelerated Degradation Protocols (ADPs): Examination of Platinum Dissolution and Carbon Corrosion in Half-Cell Tests. J. Electrochem. Soc. 2016, 163 (14), F1510-F1514. https://doi.org/10.1149/2.0731614jes.

(9) Geiger, S.; Kasian, O.; Ledendecker, M.; Pizzutilo, E.; Mingers, A. M.; Fu, W. T.; DiazMorales, O.; Li, Z.; Oellers, T.; Fruchter, L.; Ludwig, A.; Mayrhofer, K. J. J.; Koper, M. T. M.; Cherevko, S. The Stability Number as a Metric for Electrocatalyst Stability Benchmarking. Nat. Catal. 2018, 1 (7), 508-515. https://doi.org/10.1038/s41929-018-00856.

(10) Mayrhofer, K. J. J.; Meier, J. C.; Ashton, S. J.; Wiberg, G. K. H.; Kraus, F.; Hanzlik, M.; Arenz, M. Fuel Cell Catalyst Degradation on the Nanoscale. Electrochem. commun. 2008, 10 (8), 1144-1147. https://doi.org/10.1016/j.elecom.2008.05.032.

(11) Zana, A.; Speder, J.; Reeler, N. E. A.; Vosch, T.; Arenz, M. Investigating the Corrosion of High Surface Area Carbons during Start/Stop Fuel Cell Conditions: A Raman Study. Electrochim. Acta 2013, 114, 455-461. https://doi.org/10.1016/j.electacta.2013.10.097.

(12) Ohma, A.; Shinohara, K.; Iiyama, A.; Yoshida, T.; Daimaru, A. Membrane and Catalyst Performance Targets for Automotive Fuel Cells by FCCJ Membrane, Catalyst, MEA WG. ECS Trans. 2011, 41 (1), 775-784. https://doi.org/10.1149/1.3635611.

(13) Schröder, J.; Quinson, J.; Kirkensgaard, J.; Alinejad, S.; Mints, V.; Jensen, K.; Arenz, M. A New Approach to Probe the Degradation of Fuel Cell Catalysts Under Realistic Conditions: Combining Tests in a Gas Diffusion Electrode Setup with Small Angle X-Ray Scattering. $J$. Electrochem. Soc. 2020. https://doi.org/10.26434/chemrxiv.12263804.

(14) Meier, J. C.; Galeano, C.; Katsounaros, I.; Topalov, A. A.; Kostka, A.; Schüth, F.; Mayrhofer, K. J. J. Degradation Mechanisms of Pt/C Fuel Cell Catalysts under Simulated Start-Stop Conditions. ACS Catal. 2012, 2 (5), 832-843. https://doi.org/10.1021/cs300024h.

(15) Cherevko, S.; Kulyk, N.; Mayrhofer, K. J. J. Durability of Platinum-Based Fuel Cell Electrocatalysts: Dissolution of Bulk and Nanoscale Platinum. Nano Energy 2016, 29, 275298. https://doi.org/10.1016/j.nanoen.2016.03.005.

(16) Hartl, K.; Hanzlik, M.; Arenz, M. IL-TEM Investigations on the Degradation Mechanism of 
Pt/C Electrocatalysts with Different Carbon Supports. Energy Environ. Sci. 2011, 4 (1), 234 238. https://doi.org/10.1039/C0EE00248H.

(17) Hodnik, N.; Zorko, M.; Bele, M.; Hočevar, S.; Gaberšček, M. Identical Location Scanning Electron Microscopy: A Case Study of Electrochemical Degradation of PtNi Nanoparticles Using a New Nondestructive Method. J. Phys. Chem. C 2012, 116 (40), 21326-21333. https://doi.org/10.1021/jp303831c.

(18) Zana, A.; Speder, J.; Roefzaad, M.; Altmann, L.; Baumer, M.; Arenz, M.; Bäumer, M.; Arenz, M. Probing Degradation by IL-TEM: The Influence of Stress Test Conditions on the Degradation Mechanism. J. Electrochem. Soc. 2013, 160 (6), F608-F615. https://doi.org/10.1149/2.078306jes.

(19) Arenz, M.; Zana, A. Fuel Cell Catalyst Degradation: Identical Location Electron Microscopy and Related Methods. Nano Energy 2016, 29, 299-313. https://doi.org/10.1016/j.nanoen.2016.04.027.

(20) Alinejad, S.; Inaba, M.; Schröder, J.; Du, J.; Quinson, J.; Zana, A.; Arenz, M. Testing Fuel Cell Catalysts under More Realistic Reaction Conditions: Accelerated Stress Tests in a Gas Diffusion Electrode Setup. J. Phys. Energy 2020, 2 (2), 024003. https://doi.org/10.1088/2515-7655/ab67e2.

(21) Schonvogel, D.; Hülstede, J.; Wagner, P.; Kruusenberg, I.; Tammeveski, K.; Dyck, A.; Agert, C.; Wark, M. Stability of Pt Nanoparticles on Alternative Carbon Supports for Oxygen Reduction Reaction. J. Electrochem. Soc. 2017, 164 (9), F995. https://doi.org/10.1149/2.1611709JES.

(22) Souza, N. E.; Bott-Neto, J. L.; Rocha, T. A.; da Silva, G. C.; Teixeira-Neto, E.; Gonzalez, E. R.; Ticianelli, E. A. Support Modification in Pt/C Electrocatalysts for Durability Increase: A Degradation Study Assisted by Identical Location Transmission Electron Microscopy. Electrochim. Acta 2018, 265, 523-531. https://doi.org/10.1016/J.ELECTACTA.2018.01.180.

(23) Lafforgue, C.; Maillard, F.; Martin, V.; Dubau, L.; Chatenet, M. Degradation of CarbonSupported Platinum-Group-Metal Electrocatalysts in Alkaline Media Studied by in Situ Fourier Transform Infrared Spectroscopy and Identical-Location Transmission Electron Microscopy. ACS Catal. 2019, 5613-5622. https://doi.org/10.1021/acscatal.9b00439.

(24) Yu, H.; Zachman, M.; Myers, D.; Mukundan, R.; Zhang, H.; Zelenay, P.; Neyerlin, K.; Cullen, D. Elucidating Fuel Cell Catalyst Degradation Mechanisms by Identical-Location Transmission Electron Microscopy. Microsc. Microanal. 2021, 27 (S1), 974-976. https://doi.org/10.1017/S1431927621003706.

(25) Speder, J.; Zana, A.; Spanos, I.; Kirkensgaard, J. J. K. K.; Mortensen, K.; Hanzlik, M.; 
Arenz, M. Comparative Degradation Study of Carbon Supported Proton Exchange Membrane Fuel Cell Electrocatalysts - The Influence of the Platinum to Carbon Ratio on the Degradation Rate. J. Power Sources 2014, 261, 14-22.

https://doi.org/10.1016/j.jpowsour.2014.03.039.

(26) Inaba, M.; Quinson, J.; Arenz, M. PH Matters: The Influence of the Catalyst Ink on the Oxygen Reduction Activity Determined in Thin Film Rotating Disk Electrode Measurements. J. Power Sources 2017, 353, 19-27. https://doi.org/10.1016/j.jpowsour.2017.03.140.

(27) Quinson, J.; Inaba, M.; Neumann, S.; Swane, A. A.; Bucher, J.; Simonsen, S. B.; Theil Kuhn, L.; Kirkensgaard, J. J. K.; Jensen, K. M. Ø.; Oezaslan, M.; Kunz, S.; Arenz, M. Investigating Particle Size Effects in Catalysis by Applying a Size-Controlled and Surfactant-Free Synthesis of Colloidal Nanoparticles in Alkaline Ethylene Glycol: Case Study of the Oxygen Reduction Reaction on Pt. ACS Catal. 2018, 8 (7), 6627-6635. https://doi.org/10.1021/acscatal.8b00694.

(28) Schröder, J.; Quinson, J.; Mathiesen, J. K.; Kirkensgaard, J. J. K.; Alinejad, S.; Mints, V. A.; Jensen, K. M. $\emptyset$.; Arenz, M. A New Approach to Probe the Degradation of Fuel Cell Catalysts under Realistic Conditions: Combining Tests in a Gas Diffusion Electrode Setup with Small Angle X-Ray Scattering. J. Electrochem. Soc. 2020, 167 (13), 134515. https://doi.org/10.1149/1945-7111/abbdd2.

(29) Alinejad, S.; Quinson, J.; Schröder, J.; Kirkensgaard, J. J. K.; Arenz, M. Carbon-Supported Platinum Electrocatalysts Probed in a Gas Diffusion Setup with Alkaline Environment: How Particle Size and Mesoscopic Environment Influence the Degradation Mechanism. ACS Catal. 2020, 10 (21), 13040-13049. https://doi.org/10.1021/acscatal.0c03184.

(30) Binninger, T.; Garganourakis, M.; Han, J.; Patru, A.; Fabbri, E.; Sereda, O.; Kötz, R.; Menzel, A.; Schmidt, T. J. Particle-Support Interferences in Small-Angle X-Ray Scattering from Supported-Catalyst Materials. Phys. Rev. Appl. 2015, 3 (2), 024012(6). https://doi.org/10.1103/PhysRevApplied.3.024012.

(31) Ehelebe, K.; Seeberger, D.; Paul, M. T. Y.; Thiele, S.; Mayrhofer, K. J. J.; Cherevko, S. Evaluating Electrocatalysts at Relevant Currents in a Half-Cell: The Impact of Pt Loading on Oxygen Reduction Reaction. J. Electrochem. Soc. 2019, 166 (16), F1259-F1268. https://doi.org/10.1149/2.0911915jes.

(32) Speder, J.; Zana, A.; Spanos, I.; Kirkensgaard, J. J. K.; Mortensen, K.; Hanzlik, M.; Arenz, M. Comparative Degradation Study of Carbon Supported Proton Exchange Membrane Fuel Cell Electrocatalysts - The Influence of the Platinum to Carbon Ratio on the Degradation 
Rate. J. Power Sources 2014, 261, 14-22. https://doi.org/10.1016/j.jpowsour.2014.03.039.

Quinson, J.; Röefzaad, M.; Deiana, D.; Hansen, T. W.; Wagner, J. B.; Nesselberger, M.; Crampton, A. S.; Ridge, C. J.; Schweinberger, F. F.; Heiz, U.; Arenz, M. Electrochemical Stability of Subnanometer Pt Clusters. Electrochim. Acta 2018, 277, 211-217. https://doi.org/10.1016/j.electacta.2018.04.211.

(34) Martens, I.; Chattot, R.; Rasola, M.; Blanco, M. V.; Honkimäki, V.; Bizzotto, D.; Wilkinson, D. P.; Drnec, J. Probing the Dynamics of Platinum Surface Oxides in Fuel Cell Catalyst Layers Using in Situ X-Ray Diffraction. ACS Appl. Energy Mater. 2019, 2 (11), 7772-7780. https://doi.org/10.1021/acsaem.9b00982.

(35) Park, Y.-C.; Kakinuma, K.; Uchida, M.; Tryk, D. A.; Kamino, T.; Uchida, H.; Watanabe, M. Investigation of the Corrosion of Carbon Supports in Polymer Electrolyte Fuel Cells Using Simulated Start-up/Shutdown Cycling. Electrochim. Acta 2013, 91, 195-207. https://doi.org/10.1016/j.electacta.2012.12.082.

(36) Yarlagadda, V.; McKinney, S. E.; Keary, C. L.; Thompson, L.; Zulevi, B.; Kongkanand, A. Preparation of PEMFC Electrodes from Milligram-Amounts of Catalyst Powder. $J$. Electrochem. Soc. 2017, 164 (7), F845-F849. https://doi.org/10.1149/2.1461707jes.

(37) Martens, I.; Vamvakeros, A.; Martinez, N.; Chattot, R.; Pusa, J.; Blanco, M. V.; Fisher, E. A.; Asset, T.; Escribano, S.; Micoud, F.; Starr, T.; Coelho, A.; Honkimäki, V.; Bizzotto, D.; Wilkinson, D. P.; Jacques, S. D. M.; Maillard, F.; Dubau, L.; Lyonnard, S.; Morin, A.; Drnec, J. Imaging Heterogeneous Electrocatalyst Stability and Decoupling Degradation Mechanisms in Operating Hydrogen Fuel Cells. ACS Energy Lett. 2021, 2742-2749. https://doi.org/10.1021/ACSENERGYLETT.1C00718.

(38) Schröder, J.; Quinson, J.; Mathiesen, J. K.; Kirkensgaard, J. J. K.; Alinejad, S.; Mints, V. A.; Jensen, K. M. Ø.; Arenz, M. A New Approach to Probe the Degradation of Fuel Cell Catalysts under Realistic Conditions: Combining Tests in a Gas Diffusion Electrode Setup with Small Angle X-Ray Scattering. J. Electrochem. Soc. 2020, 167 (13), 134515. https://doi.org/10.1149/1945-7111/abbdd2.

(39) Speder, J.; Altmann, L.; Roefzaad, M.; Bäumer, M.; Kirkensgaard, J. J. K. K.; Mortensen, K.; Arenz, M. Pt Based PEMFC Catalysts Prepared from Colloidal Particle Suspensions - a Toolbox for Model Studies. Phys. Chem. Chem. Phys. 2013, 15 (10), 3602. https://doi.org/10.1039/c3cp50195g.

(40) Schröder, J.; Mints, V. A.; Bornet, A.; Berner, E.; Fathi Tovini, M.; Quinson, J.; Wiberg, G. K. H.; Bizzotto, F.; El-Sayed, H. A.; Arenz, M. The Gas Diffusion Electrode Setup as Straightforward Testing Device for Proton Exchange Membrane Water Electrolyzer 
Catalysts. JACS Au 2021, 1 (3), 247-251. https://doi.org/10.1021/jacsau.1c00015.

(41) Zemb, T.; Lindner, P. Neutron, X-Rays and Light. Scattering Methods Applied to Soft Condensed Matter, 1st Editio.; Zemb, T., Lindner, P., Eds.; Elsevier: Amsterdam, 2002.

(42) Kajiwara, K.; Hiragi, Y. Chapter 6 Structure Analysis by Small-Angle X-Ray Scattering. Anal. Spectrosc. Libr. 1996, 7 (C), 353-404. https://doi.org/10.1016/S0926-4345(96)800071 .

(43) Garcia, P. R. A. F.; Prymak, O.; Grasmik, V.; Pappert, K.; Wlysses, W.; Otubo, L.; Epple, M.; Oliveira, C. L. P. An in Situ SAXS Investigation of the Formation of Silver Nanoparticles and Bimetallic Silver-Gold Nanoparticles in Controlled Wet-Chemical Reduction Synthesis. Nanoscale Adv. 2020, 2 (1), 225-238. https://doi.org/10.1039/C9NA00569B.

(44) Toby, B. H.; Von Dreele, R. B. GSAS-II : The Genesis of a Modern Open-Source All Purpose Crystallography Software Package. J. Appl. Crystallogr. 2013, 46 (2), 544-549. https://doi.org/10.1107/S0021889813003531.

(45) Martens, I.; Vamvakeros, A.; Chattot, R.; Blanco, M. V.; Rasola, M.; Pusa, J.; Jacques, S. D. M.; Bizzotto, D.; Wilkinson, D. P.; Ruffmann, B.; Heidemann, S.; Honkimäki, V.; Drnec, J. X-Ray Transparent Proton-Exchange Membrane Fuel Cell Design for in Situ Wide and Small Angle Scattering Tomography. J. Power Sources 2019, 437, 226906. https://doi.org/10.1016/j.jpowsour.2019.226906.

(46) Kregar, A.; Kravos, A.; Katrašnik, T. Methodology for Evaluation of Contributions of Ostwald Ripening and Particle Agglomeration to Growth of Catalyst Particles in PEM Fuel Cells. Fuel Cells 2020, 20 (4), 487-498. https://doi.org/10.1002/fuce.201900208.

(47) Shao-Horn, Y.; Sheng, W. C.; Chen, S.; Ferreira, P. J.; Holby, E. F.; Morgan, D. Instability of Supported Platinum Nanoparticles in Low-Temperature Fuel Cells. Top. Catal. 2007, 46 (3-4), 285-305. https://doi.org/10.1007/s11244-007-9000-0. 